\title{
Perspectives provided by leopard and other cat genomes: how diet determined the evolutionary history of carnivores, omnivores, and herbivores
}

\author{
Soonok Kim ${ }^{1, *}$, Yun Sung Cho ${ }^{2,3}$, Jong Bhak ${ }^{2,3, *}$, Stephen J. O’Brian ${ }^{4,5}$ E Joo-Hong Yeo ${ }^{1}$ \\ ${ }^{1}$ Biological and Genetic Resources Assessment Division, National Institute of Biological Resources, Incheon 22689, ${ }^{2}$ The Genomics \\ Institute, Ulsan National Institute of Science and Technology (UNIST), ${ }^{3}$ Department of Biomedical Engineering, School of Life Sciences, \\ Ulsan National Institute of Science and Technology (UNIST), Ulsan 44919, Korea, ${ }^{4}$ Theodosius Dobzhansky Center for Genome \\ Bioinformatics, St. Petersburg State University, St. Petersburg 199004, Russia, ${ }^{5}$ Oceanographic Center, 8000 N. Ocean Drive, Nova \\ Southeastern University, Ft Lauderdale, Florida 33004, USA
}

Recent advances in genome sequencing technologies have enabled humans to generate and investigate the genomes of wild species. This includes the big cat family, such as tigers, lions, and leopards. Adding the first high quality leopard genome, we have performed an in-depth comparative analysis to identify the genomic signatures in the evolution of felid to become the top predators on land. Our study focused on how the carnivore genomes, as compared to the omnivore or herbivore genomes, shared evolutionary adaptations in genes associated with nutrient metabolism, muscle strength, agility, and other traits responsible for hunting and meat digestion. We found genetic evidence that genomes represent what animals eat through modifying genes. Highly conserved genetically relevant regions were discovered in genomes at the family level. Also, the Felidae family genomes exhibited low levels of genetic diversity associated with decreased population sizes, presumably because of their strict diet, suggesting their vulnerability and critical conservation status. Our findings can be used for human health enhancement, since we share the same genes as cats with some variation. This is an example

*Corresponding authors. Soonok Kim, E-mail: sokim90@korea.kr, Jong Bhak, E-mail: jongbhak@genomics.org

https://doi.org/10.5483/BMBRep.2017.50.1.002

Received 2 January 2017

Keywords: Dietary adaptation, Evolution, Felidae, Leopard, Wild species genome

Abbreviations: CBD, Convention on Biological Diversity; PSMC, Pairwise sequentially Markovian coalescent; SNV, Single nucleotide variation

Perspective to: Soonok Kim et al (2016), Comparison of carnivore, omnivore, and herbivore mammalian genomes with a new leopard assembly. Genome Biology, Nov. 2; 17:211. doi: 10.1186/s13059016-1071-4. how wildlife genomes can be a critical resource for human evolution, providing key genetic marker information for disease treatment. [BMB Reports 2017; 50(1): 3-4]

Since the Convention on Biological Diversity (CBD) was enforced in 1993, the conservation and sustainable use of biodiversity has become an essential issue for the survival of living entities, including humans, in the rapidly changing current ecosystems. Biodiversity traditionally includes species diversity, genetic diversity, and ecosystem diversity. In addition to these components, genomic diversity has recently been added as one of the fundamental layers of biodiversity.

Recent advances in genome sequencing technologies and the resulting decrease in cost assisted by the refinement of bioinformatics tools to interpret genomic codes made genomics readily available to biodiversity researches in non-model, wild species. The genome sequences of wild animal species are rapidly being accumulated, providing rich resources for the study of adaptation, trait evolution, species divergence, and population structure analyses. Currently, more than 120 genome assemblies and many more whole genome re-sequencing data are available for the mammalian taxa. These data will be used for furthering conservation efforts and for good management practices of endangered wild species.

Felidae, the family of cats, includes the most iconic and much threatened wild species such as the tiger, lion, cheetah, and leopard. Felidae species are the top predators and eat only meat to survive. As a hyper-carnivore, the felids have acquired several key diet-related traits such as digestive enzymes, shortened digestive tracts, and alteration of taste bud sensitivities to sugar. This extreme genetic adaptation endows us to generate invaluable insight and practical bio-markers in the future, for human disease and health studies as a genome diversity resource. The morphology of cats is highly adapted for hunting, powered by flexible bodies, fast reflexes, and strong muscular limbs. They also possess highly developed 
senses of night vision, hearing, and smell. The leopard, Panthera pardus, is among the most widely distributed big cats, inhabiting from Africa to Russian Far East. Of the nine genetically distinguished subspecies, the Amur leopard faces the most serious threat of extinction, with only 60-70 individuals remaining in the wild, mostly due to the increasing human population expansion, habitat loss, hunting, and poaching.

Felidae genomes are well-studied from a genomic perspective. Genome assemblies have been available for four species in Felidae, i.e., domestic cat (Felis catus), tiger (Panthera tigris), cheetah (Acinonyx jubatus), and lion ( $P$. leo). In addition, whole genome sequences for snow leopard $(P$. uncia) and additional individuals of tiger and lion have also been released.

We recently produced and added the first reference of a leopard genome to increase the number of high quality cat genome repertoire, from a captive Amur leopard from Daejeon Zoo of Korea. We sequenced it to a $310 \times$ average depth using the Illumina HiSeq platforms, and assembled the sequences into 50,400 scaffolds (N50 length of $21.7 \mathrm{Mb}$ ) resulting in 2.58 Gb length, which is the highest quality among the big cat genomes. A total of 19,043 protein-coding genes were annotated, with $39.04 \%$ of the genome being various repeats. Additionally, we produced two whole genome re-sequences at the depth of $35 \times$ from free ranging wild leopards obtained from the "land of leopard" National Park in Far East Russia, and one wild leopard cat (Prionailurus bengalensis) from South Korea.

On an average, the genetic diversity of Felidae was very low, reflecting the small population size with a high propensity for extinction, as assessed with heterozygous single nucleotide variation (SNV) rates against the domestic cat genome. The genetic diversities of three leopards were similar to those of the snow leopard, cheetah, and white lion, which have extremely low diversity due to habitat isolation or inbreeding. Using the pairwise sequentially Markovian coalescent (PSMC) model inference, we calculated the demographic history of leopards, which revealed a rapid decline of effective population size since its divergence 10 million years ago, with traces of a severe genetic bottleneck between two million to $900 \mathrm{~K}$ years ago.

Extensive comparative analyses with publicly available mammalian genomes were conducted to identify genomic signatures of Felidae evolution, with the main focus on its hyper-carnivorous life style. At first, evolutionary aspects were analyzed at the amino acid level, using 8,648 orthologous gene families among the 18 mammalian genome assemblies of three dietary groups, i.e., eight carnivores (leopard, cat, tiger, cheetah, lion, polar bear, killer whale, and Tasmanian devil), five omnivores (human, mouse, dog, pig, and opossum), and five herbivores (giant panda, cow, horse, rabbit, and elephant). Felidae or carnivore specific gene expansion and contraction, positively selected genes, and convergent amino acid changes reflected its phenotypes appropriately, in which the gene families associated with muscle activities were among the expanded, while those with starch metabolism and detoxification of plant-derived toxicants were contracted. Molecular signatures for adaptation were further verified from the search of highly conserved regions across species. Certain homozygous genomic regions were strongly conserved among 14 individuals of the seven species within Felidae, stretched 1.13 $\mathrm{Gb}$ in length containing genes functionally enriched in sensory perception of light stimulus and synaptic transmission, reflecting the characteristic features of Felids such as eminent night vision and fast reflexes. Although these genomic signatures are not validated experimentally, the genetic variation information clearly indicates evolutionary adaptation of the family. A far reaching conclusion of our study is that carnivory is a rather special adaptation, as compared to omnivory and herbivory. It shows that while cows and humans can eat plants and meat if necessary, cats do not have an option for consuming plants. An investigation we did not undertake was study of the microflora of guts of the three different groups. It is likely that there are microbiomes that co-evolved with the hosts to digest plants and meat. This hologenome, the combination of the host and symbionant genomes, can provide another layer of insight on how animals evolved to extreme dietary selection. In future, when all the major kingdom's reference genomes are available, it will be necessary to iterate our study to include insects, fish, birds, reptiles, and amphibians, to enable a complete picture of genomic diversity of dietary adaptation.

\section{ACKNOWLEDGEMENTS}

This work was supported by a grant from National Institute of Biological Resources of Korea (NIBR201603104), and by the Research Fund (1.150014.01) of Ulsan National Institute of Science \& Technology (UNIST). 